\title{
Uma Tipologia de Crianças e Adolescentes em Situação de Rua Baseada na Análise de Aglomerados (Cluster Analysis)
}

\author{
Raul Aragão Martins ${ }^{1}$ \\ Universidade Estadual Paulista, São José do Rio Preto
}

\begin{abstract}
Resumo
Crianças e adolescentes em situação de rua vêm sendo estudados de forma sistemática desde a década de 1970. Os primeiros estudos procuraram quantificar esta população, e posteriormente, para melhor conhecê-los, surgiram tipologias baseadas em um critério, como tempo de permanência na rua, ou dois, tempo mais vínculo familiar. Com a finalidade de oferecer um procedimento de classificação, e uma tipologia, este estudo aplicou a análise de aglomerados Cluster Analysis a dois grupos. O primeiro composto de praticamente todas as crianças e os adolescentes em situação de rua no centro de uma cidade do interior do Estado de São Paulo, totalizando 67 pessoas, e o segundo uma amostra de 31 crianças e adolescentes, de dois bairros da mesma cidade, participantes de grupos de rua. Para o primeiro grupo foram selecionados 12 critérios e para o segundo, 7, que resultaram, para ambos grupos, em 3 aglomerados distintos. Foram também comparados estes resultados com censo de crianças de rua realizado há 4 anos. Resultados mostraram que este tipo de análise é útil na classificação destas crianças, que por sua vez poderá subsidiar melhor planejamento e ações de políticas públicas para o setor.

Palavras-chave: Crianças de rua; tipologia; uso de drogas; análise de aglomerados.
\end{abstract}

\section{A Typology of Street Children Based on Cluster Analysis}

\begin{abstract}
Street children and teenagers have been studied in a systematic way since the seventies. The first studies tried to quantify this population, and afterwards in order to know them better, typologies appeared based on the period they spend in the streets, or both the period in the street and their family relationship. In order to offer a classification procedure, as well as a typology, this research applied cluster analysis on two groups. The first one is composed by almost all downtown street children and teenagers of a city in the state of São Paulo, with 67 children and teenagers in total, and the second a sample of 31 children and teenagers from two neighborhoods of the same city all from the street. For the first group 12 criteria were chosen and for the second group, 7, which resulted in 3 different clusters. These results were also compared with the street children's census carried out four years ago. The results showed that this type of analysis is useful for children's classification, and besides that will subsidize a better planning and public political actions for this section. Keywords: Street children; typology; drug use; cluster analysis.
\end{abstract}

Crianças ou adolescentes perambulando pelas grandes cidades é um fenômeno antigo: encontramos referências na literatura, em personagens isolados, como Oliver Twist de Charles Dickens (1956) ou em grupo, como os Capitães da Areia de Jorge Amado (1965). Geremek (1995), estudando a forma como a literatura européia, entre 1400 e 1700, retrata a vida de vagabundos e miseráveis, destaca Till Eulenspiegel, adolescente alemão que teria vivido no século XIV e seria o protótipo de personagens subseqüentes, que viviam "soltos" nas grandes cidades e subsistiam de furtos e pequenos golpes, aplicados em pessoas tolas.

Embora essa figura esteja presente em todas as cidades, principalmente nos países do terceiro mundo ou em desenvolvimento, somente após 1979 encontramos trabalhos acadêmicos tratando do tema (Ferreira, s/d; Mermet, 1995; Rizzini \& Rizzini, 1996; Valladares, 1988),

1 Endereço para correspondência: Rua Cristovão Colombo, 2265, 15054000, São José do Rio Preto, SP. Fone: (17) 221 2317, Fax: (17) 224 8692. Email:raul@edu.ibilce.unesp.br coincidentemente, esse foi o Ano Internacional da Criança, promovido pela ONU, e esse evento desencadeou, pelo menos em nosso país, uma grande mobilização popular em prol do bem-estar da criança.

Antes de vermos essa preocupação com as crianças como simples conseqüência de políticas delineadas em fóruns internacionais, como a ONU, Pilotti (1995) considera que a chegada a esse ponto deve ser compreendida como uma evolução da sociedade ocidental, que começa com o homem adulto liberando-se da gerontocracia, feudalismo e escravidão e passando, posteriormente, pela liberação feminina, com a qual a mulher passa a ser vista enquanto ser em si, e não somente como filha ou esposa.

Pilotti (1995) completa, citando Aylwin, que essa evolução passa, a partir do século XVIII, por três gerações de direitos. A primeira, chamada de "direitos da liberdade", refere-se aos direitos civis e políticos; a segunda "direitos da igualdade", corresponde aos direitos econômicos, sociais e culturais; e, finalmente, neste século, surgem os "direitos 
de terceira geração" relativos ao meio ambiente, consumidores e desenvolvimento.

A consolidação desses direitos se faz na doutrina dos direitos humanos, doutrina esta que dá sustentação à questão da cidadania. Marshall (citado em Pilotti, 1995) expõe da seguinte forma:

O conceito de cidadania compreende direitos civis, necessários para garantir a liberdade individual; direitos políticos, indispensáveis para permitir a participação do exercício do poder; e os direitos sociais, que cobrem a gama de direitos requeridos para assegurar que, dentro dos padrões de uma sociedade dada, cada indivíduo possa desfrutar da segurança oferecida pelo bemestar econômico, compartilhar plenamente a herança sóciocultural e viver digna e civilizadamente. (Pilotti, 1995, p. 21)

Em 1989, a ONU aprova a Convenção dos Direitos da Criança, colocando-a como sujeito de direito e como cidadão privilegiado, dentro do princípio da proteção integral, e nosso país, já em 1990, como decorrência de discussões em âmbito nacional, aprova o Estatuto da Criança e do Adolescente de acordo com essa nova orientação (CONDECA, 1996). Nesse contexto, veremos o que foi produzido nesses quase 20 anos de pesquisas, a evolução da definição de crianças de rua e sua origens.

Ao revisarmos estudos sobre crianças em situação de rua, encontramos, num primeiro momento, artigos falando sobre o tema em vários periódicos e relatórios técnicos (Aptekar, 1991; Carrizosa \& Poertner, 1992; Cosgrove, 1990; Lusk, 1992; Oliveira, Baizerman \& Pellet, 1992; Unicef, 1991, 1993), sendo que a maioria deles retrata países em desenvolvimento, principalmente os da América Latina.

Estes textos apresentam duas características principais: a primeira afirma que existem cerca de 80 milhões de crianças em situação de rua no mundo, 40 milhões delas vivendo na América Latina (que tem somente dez por cento da população mundial), e dessas 40 milhões, metade vivendo em nosso país. Esses números talvez tenham saído de projeções da população geral do mundo, e particularmente, da América Latina e Caribe, pois, como Pilotti (1995) expõe, o número de crianças e adolescentes, no início desta década, na América Latina e Caribe, é impressionante: são cerca de 200 milhões e representam perto de $45 \%$ da população. Deste total quase a metade vive na pobreza crítica, situação esta que Pilotti sintetiza na afirmação "a maioria das crianças é pobre e a maioria dos pobres é criança” (p. 15).

A segunda característica é a falta de uma padronização do que sejam crianças e adolescentes em situação de rua, falta esta que faz com que tenhamos os números mais variados, pois, na maioria das vezes, conta-se como crianças e adolescentes em situação de rua todas as crianças pobres. Procurando ver como estas crianças e adolescentes vêm sendo categorizadas, Martins (1996b) revisa a literatura e encontra como ponto inicial a definição elaborada pela Inter-NGO (1983, citado em Martins, 1996b) e usada, também, pelas Nações Unidas (ICCB, 1985, citado em Martins 1996b):

Uma criança de rua é qualquer menino ou menina que não alcançou a idade adulta e para quem a rua (no senso mais amplo da palavra, incluindo casas abandonadas, terrenos baldios, etc.) tornou-se sua habitual fonte de vida e que é inadequadamente protegida, supervisionada ou orientada por adultos responsáveis. (p. 36)

Em 1986 a Unicef apresenta nova tipologia composta de três itens, em que o principal ponto é o contato familiar, dessa forma, temos: a) "candidatos para a rua": crianças trabalhando na rua mas vivendo com sua família; b) "criança na rua" com suporte familiar "inadequado e/ ou esporádico"; c) "criança de rua": "funcionalmente sem suporte familiar.” Assim, temos, num primeiro momento, a simples constatação da presença de crianças em situação de rua e "fora do controle" das famílias ou adultos para, num segundo momento, começarmos a fazer uma tipologia baseada no contato familiar

Posteriormente Lusk (1992) propõe, a partir de trabalho com crianças e adolescentes de rua da cidade do Rio de Janeiro, a seguinte classificação: a) trabalhadores de rua com base familiar; b) trabalhadores de rua independentes de família; c) crianças de rua; d) crianças de famílias de rua (Lusk, 1992). Essa última definição incorpora um segundo elemento ao contato familiar, o trabalho. É reconhecido que estas crianças não estão simplesmente perambulando o dia inteiro pelas ruas, e, sim, usando este espaço para busca de renda, via trabalho. Outros autores procuram descrever as crianças e adolescentes em situação de rua, sem a preocupação de formar uma tipologia, mas salientando que a situação de pobreza, por si só, não caracteriza a condição de criança e adolescente em situação de rua (Carrizosa \& Poertner, 1992; Oliveira, Baizerman \& Pellet, 1992; Williams, 1993).

Temos como características dessas definições a sua origem, isto é, surgem em trabalhos realizados nos países em desenvolvimento, e a ênfase nas condições sociais destas sociedades. Em contraposição a essa tendência, Cosgrove (1990) considera que as primeiras definições vieram dos trabalhos norte-americanos sobre "sem-tetos" (bomeless) e andarilhos (runaways), por estes terem em comum com as crianças e adolescentes em situação de rua a interação do indivíduo e o meio ambiente social imediato. Segundo essa perspectiva, o autor propõe que crianças e adolescentes em situação de rua sejam estudadas 
baseando-se em duas dimensões que considera essenciais: a pessoa e o meio.

Essas dimensões são melhor entendidas por meio dos indicadores sociais associados a elas. Assim, temos a família, ou melhor, o nível de envolvimento familiar, como indicador de meio ambiente e comportamentos aceitos pela comunidade (adesão às normas) como indicador de pessoa. Por sua vez, cada um desses indicadores apresenta três níveis de envolvimento ou adesão. Assim, envolvimento familiar é classificado em efetivamente envolvido, inconsistentemente envolvido e sem envolvimento, e adesão às normas em adesão essencial, adesão inconsistente e não adesão. A combinação desses níveis permite chegar-se a nove tipos que incluem crianças e adolescentes em situação de rua, candidatos a crianças e adolescentes em situação de rua, sujeitos com problemas de ajustamento, pseudocrianças e adolescentes de rua e crianças e adolescentes que não são de rua.

Em contraste com as definições anteriores, em que o foco é situado nas relações sociais, a tipologia de Cosgrove (1990) é baseada no próprio sujeito, isto é, a criança em situação de rua seria resultado da combinação de envolvimento familiar pobre com a não adesão às regras aceitas pela comunidade.

Martins (1996a) realizando censo das crianças e adolescentes em situação de rua na cidade de São José do Rio Preto, São Paulo, encontra dificuldades para classificar os seus dados, usando as tipologias existentes, dessa forma faz uma crítica e apresenta uma proposta baseada na busca de renda, alimentação, local de repouso e lazer, que resultou nos seguintes tipos:

Tipo 1: Crianças e adolescentes que buscam renda para família (e/ou para seu próprio sustento) e alimentamse nas ruas, onde passam a maior parte do dia, dormem freqüentemente em casa e têm lazer no bairro em que moram ou nos logradouros públicos em que trabalham. Vínculo familiar estável.

Tipo 2: Crianças e adolescentes que buscam renda para o seu próprio sustento e/ou para família (via mendicância ou furtos), alimentam-se, dormem e têm lazer nas ruas. Têm família na cidade, fazendo visitas ocasionalmente.

Tipo 3: Crianças e adolescentes que buscam renda para o seu próprio sustento nas ruas, alimentam-se e dormem em casa, mas têm lazer constante nas ruas. Vínculo familiar estável.

Tipo 4: Crianças e adolescentes que buscam renda para sustento próprio (via mendicância ou furtos), alimentam-se, dormem e têm lazer na rua. Não têm ligação familiar.

Tipo 5: Crianças e adolescentes que não buscam renda, não se alimentam ou dormem nas ruas, mas têm lazer predominantemente no espaço público. Esta situação ocorre por três motivos principais, que podem aparecer isolada ou conjugadamente: a) pais trabalham o dia inteiro fora de casa e não têm outro adulto ou irmão mais velho para tomar conta das crianças quando estas não estão na escola; b) ambiente familiar negativo (do ponto de vista da criança); c) a residência não oferece condições para permanência da criança (vivem em barracos). Vínculo familiar estável.

O último ponto, origem destas crianças e adolescentes, pode ser melhor entendido à partir do trabalho de Aptekar (1996), que usando relatos da Unicef de 1990, mostra que existiam cerca de 369 milhões de crianças e adolescentes pobres, abaixo de 15 anos de idade, nas cidades dos países em desenvolvimento, e as contagens e censos, feitos nas grandes cidades, mostram que os números desses indivíduos que usam o espaço da rua como sobrevivência é muito pequeno (ver Rosemberg, 1994; Rizzini \& Rizzini, 1996, para revisão de estudos brasileiros e Aptekar, 1996, para estudos de outros países).

Analisando os estudos Aptekar enumera três hipóteses para a origem de crianças e adolescentes em situação de rua: a pobreza, a violência doméstica e a modernização da própria sociedade. Considera que, para compreendermos este fenômeno, precisamos ter em mente que cada hipótese, tomada isoladamente, não responde às nossas questões.

Com a publicação de trabalhos sistemáticos, sejam contagens ou censos, encontramos um número muito pequeno de crianças e adolescentes (comparado com os primeiros números), usando o espaço da rua para a busca de sustento próprio ou próprio e família (Martins, 1996a; Rizzini \& Rizzini, 1996; Rosemberg, 1994, 1996). Esse fato nos obriga a refletir a questão da pobreza como única responsável pela situação destes indivíduos. A outra hipótese explicativa tem sido a violência doméstica, aliada ou não à pobreza, violência que pode vir de pais ou responsáveis mentalmente enfermos, usuários de drogas lícitas ou ilícitas ou condições de vida desfavoráveis e por último a questão da modernidade tem sido vista pelo impacto das transformações que todas as culturas estão sofrendo, como o aumento crescente das populações das cidades, em detrimento do campo, novas tecnologias diminuindo o número de empregos.

Esse quadro mostra, por um lado, um volume considerável de conhecimento e, por outro, a necessidade de conhecer melhor o fenômeno em tela; e para tanto, propõe-se um trabalho que dê continuidade ao estudo anterior, isto é, a realização de um novo censo, estudo que permitirá conhecer melhor a evolução dessas pessoas em sua entrada, permanência e saída da situação de rua, pois os estudos têm mostrado que o problema das crianças e adolescentes em situação de rua não é uniforme 
e as várias tipologias surgiram justamente para dar conta dos vários aspectos que compõem essa população.

Mas estas tipologias são resultado de classificações feitas a partir de uma característica mais proeminente, como por exemplo, vínculo familiar ou tipo de atividade, sem levar em conta as várias facetas que estas crianças e adolescentes possuem. Revendo literatura sobre modos de classificação que envolvessem muitos objetos, com muitos atributos, a ferramenta mais usada, não só na Psicologia, como em outras áreas do conhecimento, notadamente na Biologia, é a Análise de Aglomerados (Cluster Analysis) (Danseco \& Holden, 1998; Hair, Anderson, Tathan \& Black, 1998; Manzato, 1983; Pereira, 1999; Romesburg, 1990). Esse procedimento procura agrupar objetos baseados nos seus atributos, podendo ser usada de modo exploratório ou confirmatório e apresenta as duas vantagens: não existência de pressupostos, como os exigidos para uma análise fatorial e a não necessidade de um número elevado de casos. Neste último ponto, temos, por exemplo, o estudo de Pereira e Saes (1995), que analisaram sete variáveis de desempenho científico em sete Institutos de Pesquisa.

Este estudo tem dois objetivos. O primeiro fazer uma análise exploratória de dois grupos de crianças e adolescentes, tidas como "crianças de rua". O primeiro grupo permanece mais na região central e o segundo em dois bairros populares, na periferia de uma cidade de porte médio do interior do estado de São Paulo. Consideramos que o desenvolvimento de uma tipologia baseada em critérios mais precisos e acessíveis a outros pesquisadores contribua para um melhor conhecimento destas pessoas e, por sua vez, a definição e aplicação de políticas de atendimento mais adequadas. $\mathrm{O}$ segundo objetivo é levantar, entre os entrevistados neste censo, os que já tinham sido cadastrados no primeiro estudo (Martins, 1996a), para que tenhamos uma idéia do tempo de permanência destas pessoas na situação de rua.

\section{Método}

O levantamento da situação de crianças e adolescentes em situação de rua tem sido feito basicamente por meio de duas técnicas, a contagem simples e o censo. $\mathrm{Na}$ primeira, a contagem simples, a cidade é dividida em áreas onde pessoas treinadas contam, simultaneamente, as crianças e adolescentes em situação de rua em suas áreas, não as identificando. As áreas e os roteiros são definidos previamente, por meio de pré-estudos. Como também é definido previamente o horário da contagem, temos um retrato estático da situação. Uma forma de ampliar os resultados é fazerem-se várias contagens, em dias da semana e horários diferenciados, mas tendo o cuidado de lembrar que esses resultados não podem ser somados.

$\mathrm{Na}$ segunda técnica, o censo, as crianças e adolescentes são identificados e entrevistados, para coleta de dados, por pessoas treinadas, que ficam mais tempo na rua. Com essa abordagem, embora mais demorada, podemos obter dados mais precisos.

A escolha de uma dessas técnicas passa, em primeiro lugar, pelo tamanho da cidade a ser pesquisada e, em segundo, pelo tipo de política que se pretende implantar na cidade em relação a crianças e adolescentes em situação de rua. Desta forma, em função da área geográfica ocupada pela cidade e sua população, assim como a existência de inúmeros trabalhos sendo realizados com crianças e adolescentes carentes, a contagem simples não traria dados importantes para o conhecimento desse segmento. Sendo assim, por ser uma cidade de porte médio, comporta adequadamente fazer um censo, onde, com um custo pequeno podemos ter dados fidedignos.

A forma de tratar uma questão tão complexa como essa, a saber, a de fazer-se um levantamento das crianças e adolescentes em situação de rua, está parcialmente estabelecida na bibliografia científica (Martins, 1996a); porém o que não deve ser menosprezado são as características de cada situação em particular. Assim trabalhamos com uma equipe formada por recenceadores experientes, composta por seis pessoas que já vinham atuando com esta população. Desta forma não houve necessidade de treinamento específico.

Para entrevistarmos as crianças e adolescentes, em primeiro lugar pedimos autorização ao Conselho Municipal dos Direitos da Criança e do Adolescente, o que foi concedido, e usamos o instrumento desenvolvido no primeiro censo (Martins, 1996a), acrescido de ítens considerados relevantes para este levantamento, que resultaram em quatro campos de informações. O primeiro, chamado de identificação geral, com dados do local, dia e hora da entrevista e nome do entrevistador. O segundo, identificação do participante, com o nome, idade, sexo e cor do participante. O terceiro, dados gerais, com levantamento da atividade realizada na hora da entrevista, tempo de rua, motivo para estar na rua, dados escolares, profissionais, procedência da família, participação em programas sociais, uso de drogas, local de alimentação e repouso, retorno para casa e formas de brincar. Finalmente o último campo sobre dados familiares.

\section{Resultados}

Este estudo embora tenha tido como foco principal o levantamento das crianças e adolescentes em situação 
de rua em São José do Rio Preto foram, também, cadastrados participantes de quatro programas voltados para populações sem supervisão adequada de adultos, programas pós-escola, e que embora compartilhem algumas características das crianças em situação de rua, elas têm um perfil diferenciado, que pretendo estar analisando em outro artigo.

Após esta introdução foi feita uma organização das variáveis, tendo em vista dois pontos. O primeiro que elas fossem semelhantes às variáveis estudadas em outras pesquisas e o segundo que permitissem uma delimitação da situação de vivência dos participantes. Desta forma, retiramos do campo identificação do participante as variáveis idade (crianças e adolescentes), sexo (masculino e feminino) e cor (pardos/negros e brancos). Dos dados gerais compactamos as informações atividade e motivo para estar na rua na categoria atividade (trabalho de ambulante, engraxate ou panfleteiro e atividade de esmolar, perambular, brincar ou dormir na rua); tempo de rua (menor ou igual a seis meses e maior que seis meses); dados escolares resultaram nas categorias sobre freqüência à escola no ano de 1997 e 1998 e estar alfabetizado; local de alimentação, local de repouso e retorno para casa resultaram na categoria retorno diário para casa (sim ou não); participar de programa de apoio à criança e ou adolescente (sim ou não); uso de drogas (sim ou não) e procedência da família (da cidade local do estudo ou de outras cidades). Não utilizamos a variável brincar por dificuldade na coleta desta informação, assim como as informações do campo com dados familiares, que estão tendo uma análise a parte.

Após a organização das variáveis separamos os participantes em dois grupos: os entrevistados na região central da cidade e os dos bairros próximos às suas residências. Esta divisão baseou-se no tipo de uso do espaço da rua e na visão que estas crianças e adolescentes tinham de si e de suas atividades, isto é, os participantes dos bairros não se viam como "de rua". Finalmente, consideramos que embora contando com um número pequeno de participantes (quando comparado com os dados de cidades com mais de um milhão de habitantes), consideramos que estes dados venham a contribuir para uma melhor compreensão da dinâmica destas pessoas.

Em termos numéricos entrevistamos 67 crianças e adolescentes em situação de rua, que passamos a chamar de grupo "Rua" e 31 nos bairros, que nomeamos "Bairro". Estes participantes são inicialmente apresentados por sexo, cor e idade. A Tabela 1 mostra o grupo "Rua", onde encontramos um predomínio do sexo masculino: 93\% dos casos; dos adolescentes: $84 \%$ e os com cor branca: $60 \%$. Dados, que com exceção da cor, correspondem aos encontrados em outras cidades.

No grupo "Bairro" encontramos $65 \%$ de pessoas do sexo masculino, $68 \%$ da cor preta ou parda e $94 \%$ de adolescentes (Tabela 2). Em relação ao grupo "Rua" se igualam apenas na idade, a maioria é adolescente, nos outros dois pontos: cor e sexo, há uma predominância de negros e pardos, e o predomínio do sexo masculino não é tão grande.

Como já mostramos a identificação de diferentes tipos de crianças e adolescentes em situação de rua tem sido alvo de vários estudos. Com estas pessoas sendo classificadas por um ou dois critérios mais salientes, como vínculo familiar ou tempo de permanência na rua, criando categorias simplistas, que ao mesmo tempo que fornece base para algum tipo de intervenção, não fornece muita informação, assim realizamos uma Análise de Aglomerado seguindo os passos propostos por Hair e colaboradores (1998) e Romesburg (1990).

Tabela 1

Grupo Rua por Sexo, Cor e Idade

\begin{tabular}{|c|c|c|c|c|c|c|c|}
\hline \multicolumn{4}{|c|}{ Feminino } & \multicolumn{3}{|c|}{ Masculino } & \multirow[b]{2}{*}{ Tota } \\
\hline Cor & Branca & Preta & Parda & Branca & Preta & Parda & \\
\hline \multicolumn{8}{|l|}{ Idade } \\
\hline 9 & 0 & 0 & 0 & 1 & 0 & 0 & 1 \\
\hline 10 & 0 & 0 & 0 & 2 & 0 & 1 & 3 \\
\hline 11 & 1 & 0 & 0 & 2 & 2 & 2 & 7 \\
\hline 12 & 1 & 0 & 0 & 5 & 0 & 3 & 9 \\
\hline 13 & 0 & 0 & 0 & 9 & 2 & 1 & 12 \\
\hline 14 & 0 & 0 & 0 & 8 & 2 & 4 & 14 \\
\hline 15 & 1 & 0 & 0 & 1 & 1 & 1 & 4 \\
\hline 16 & 2 & 0 & 0 & 3 & 3 & 3 & 11 \\
\hline 17 & 0 & 0 & 0 & 4 & 1 & 1 & 6 \\
\hline Total & 5 & 0 & 0 & 35 & 11 & 16 & 67 \\
\hline
\end{tabular}


Tabela 2

Grupo Bairro por Sexo, Cor e Idade

\begin{tabular}{cccccccccccc}
\hline \multicolumn{10}{c}{ Feminino } \\
\hline Cor & 1 & 2 & 3 & 4 & 5 & 1 & 2 & 3 & 4 & 5 & Tatal \\
\hline Idade & & & & & & & & & & & 2 \\
10 & 0 & 0 & 0 & 0 & 0 & 0 & 0 & 2 & 0 & 0 & 2 \\
12 & 0 & 0 & 0 & 0 & 0 & 0 & 2 & 0 & 0 & 0 & 7 \\
13 & 1 & 1 & 4 & 0 & 0 & 0 & 1 & 0 & 0 & 0 & 7 \\
14 & 0 & 0 & 1 & 0 & 0 & 2 & 2 & 2 & 0 & 0 & 6 \\
15 & 0 & 1 & 0 & 0 & 1 & 2 & 0 & 2 & 0 & 0 & 3 \\
16 & 0 & 1 & 0 & 0 & 0 & 0 & 0 & 0 & 2 & 0 & 4 \\
17 & 0 & 0 & 1 & 0 & 0 & 1 & 0 & 1 & 0 & 1 & 4 \\
\hline Total & 1 & 3 & 6 & 0 & 1 & 5 & 5 & 7 & 2 & 1 & 31 \\
\hline
\end{tabular}

Cor: 1 = branca; $2=$ preta; $3=$ parda; $4=$ amarela; $5=$ não-especificada

O primeiro refere-se aos objetivos da análise; aqui neste caso é o de compreender melhor como se agrupam as crianças e adolescentes entrevistados. Baseado neste objetivo trabalhamos com as 12 variáveis que consideramos como discriminadoras da situação de rua. Estas variáveis foram categorizadas em termos dicotômicos, por considerarmos uma forma mais simples de visualizarmos estes participantes, atribuindo o código "1" a presença e "0" para a ausência

Tabela 3

Nome das Variáveis e sua Discriminação do atributo que caracteriza a criança e ou adolescente em situação de rua, como por exemplo, esperamos que eles tenham freqüentado a escola no ano de 1997, mas a tenham abandonado no de 1998 (Tabela 3).

O segundo passo refere-se ao delineamento da pesquisa, que envolve a detecção de valores "intrusos" (outliers), a escolha da medida de similaridade e a decisão de estandardizar ou não os dados. Quanto ao primeiro ponto Variável Código Discriminação

\begin{tabular}{|c|c|c|}
\hline Atividade & 0 & Trabalho de ambulante, engraxate ou panfleteiro \\
\hline & 1 & Atividade de esmolar, perambular, brincar ou dormir na rua \\
\hline Sexo & 0 & Feminino \\
\hline & 1 & Masculino \\
\hline Cor & 0 & Negra ou parda \\
\hline & 1 & Branca \\
\hline Idade & 0 & Crianças \\
\hline & 1 & Adolescentes \\
\hline Tempo & 0 & Tempo de rua menor ou igual a seis meses \\
\hline & 1 & Tempo de rua maior que seis meses \\
\hline Escola 97 & 0 & Não freqüentou à escola no ano de 1997 \\
\hline & 1 & Freqüentou à escola no ano de 1997 \\
\hline Escola 98 & 0 & Freqüentou à escola no ano de 1998 \\
\hline & 1 & Não freqüentou à escola no ano de 1998 \\
\hline Alfabetização & 0 & Alfabetizado \\
\hline & 1 & Não alfabetizado \\
\hline Procedência & 0 & Outras cidades \\
\hline & 1 & São José do Rio Preto \\
\hline Programas & 0 & Não participou programa de apoio à criança e/ou adolescente \\
\hline & 1 & Participou de programa de apoio à criança e/ou adolescente \\
\hline Drogas & 0 & Não é usuário de drogas \\
\hline & 1 & É usuário de drogas \\
\hline Retorno & 0 & Retorno todos os dias para dormir em sua casa \\
\hline & 1 & Não retorna todos os dias para dormir em sua casa \\
\hline
\end{tabular}


não temos valores “intrusos" por termos transformado as respostas originais em termos dicotômicos, o que já responde o terceiro ponto. A transformação das variáveis em termos de presença ou ausência se deveu a grande variedade de medidas usadas, o que recomendaria a estandardização destas medidas. A escolha da medida de similaridade recaiu sobre a size difference por ser uma das mais adequadas para dados dicotômicos e o método de aglomeração usado foi o de Ward (ver passo três).

O terceiro passo refere-se a seleção do método de aglomeração a ser usado. Em primeiro lugar optamos por um método hierárquico por não termos, a priori, uma variável que servisse de apoio para iniciar o processo de aglomeração (cluster seed). Desta forma, dentro dos métodos disponíveis, optamos pelo de Ward, por este usualmente promover ótimas soluções (Romesburg, 1990).

Realizada a análise e de posse do dendograma, partimos em busca da melhor configuração, que se evidenciou ao "cortamos a árvore" no nível de três aglomerados. Os participantes agrupados em cada um dos aglomerados mostraram distintas formas de relações com o espaço da rua (Tabela 4). Detalhando cada um, encontramos, as seguintes características:

- Aglomerado 1: chamados de "solitários" por predominar a falta de supervisão de adultos, com 27 dos casos, corresponde a 40,3\% do total, tem $96 \%$ dos seus membros usando o espaço de rua para mendigar/ perambular/brincar e usar drogas, 100\% com tempo de rua alto, abandono progressivo da escola ( $70 \%$ não foi à escola em 1997, número que passou para 89\% em 1998) resultando em $63 \%$ de analfabetos e $85 \%$ não retornando diariamente para casa.
- Aglomerado 2: chamado de "desligando-se", é o menor grupo, com 11 casos, correspondendo a 16,4\% do total, tem $64 \%$ dos seus membros usando o espaço de rua para mendigar/perambular/brincar, cerca da metade faz uso de drogas (55\%), 64\% deles com tempo de rua alto, um quadro menos acentuado de abandono progressivo da escola $(92 \%$ foram à escola no ano de 1997, caindo para $73 \%$ em 1998) resultando em um número menor de analfabetos (18\%) e 55\% não retornando diariamente para casa.

- Aglomerado 3: chamado de "trabalhadores", é o maior grupo, com 29 membros, correspondendo a 43,3\% dos casos, se caracteriza por mais da metade usar o espaço de rua como local de trabalho (55\%) e 100\% deles não se envolveram com drogas, embora $52 \%$ deles tenham tempo alto de rua. É o grupo com o mais alto índice de freqüência à escola (93\% em 1997 e 84\% em 1998), resultando na menor taxa de analfabetismo e de não retorno à casa diariamente (ambas variáveis com 7\%).

A partir das análises feitas com os dados do grupo "Rua", onde foram selecionadas 12 variáveis como caracterizadoras da situação de rua (Tabela 3), montamos uma nova matriz, com os participantes do grupo "Bairro" e com as variáveis que se aplicavam a esse grupo. Este novo conjunto, com sete variáveis, ficou composto de: sexo, cor, freqüência à escola em 1997, freqüência à escola em 1998, alfabetização, participação em programas e uso de drogas.

Com estas novas variáveis aplicamos os mesmos procedimentos de análise aos 31 participantes do grupo "Bairro", e a partir do dendograma gerado "cortamos a árvore" no nível de formação de três aglomerados, que

Tabela 4

Freqüencia e Porcentagem dos Participantes nos Aglomerados por Variáveis

\begin{tabular}{|c|c|c|c|c|c|c|}
\hline \multirow[b]{3}{*}{ Variáveis } & \multicolumn{2}{|c|}{ Aglomerado 1} & \multicolumn{2}{|c|}{ Aglomerado 2} & \multicolumn{2}{|c|}{ Aglomerado 3} \\
\hline & $f$ & $\%$ & $f$ & $\%$ & $f$ & $\%$ \\
\hline & & & & & & \\
\hline Esmolar/perambular & 26 & 96 & 7 & 64 & 13 & 45 \\
\hline Sexo masculino & 26 & 96 & 10 & 91 & 26 & 90 \\
\hline Cor - negras ou pardas & 14 & 52 & 6 & 55 & 7 & 24 \\
\hline Idade - adolescentes & 25 & 93 & 8 & 73 & 23 & 79 \\
\hline Tempo de rua alto & 27 & 100 & 7 & 64 & 15 & 52 \\
\hline Freqüência escola 97 & 8 & 30 & 9 & 92 & 27 & 93 \\
\hline Não freqüência escola 98 & 24 & 89 & 3 & 27 & 4 & 16 \\
\hline Não alfabetização & 17 & 63 & 2 & 18 & 2 & 7 \\
\hline Procedência de SJRP & 23 & 85 & 7 & 64 & 13 & 45 \\
\hline Participação em programas & 22 & 81 & 8 & 73 & 10 & 34 \\
\hline Uso de drogas & 26 & 96 & 6 & 55 & 0 & 0 \\
\hline Não retorno diário à casa & 23 & 85 & 6 & 55 & 3 & 7 \\
\hline
\end{tabular}


Tabela 5

Frequiência e Porcentagem dos Participantes nos Aglomerados por Variáveis

\begin{tabular}{|c|c|c|c|c|c|c|}
\hline & \multicolumn{2}{|c|}{ Aglomerado 1} & \multicolumn{2}{|c|}{ Aglomerado 2} & \multicolumn{2}{|c|}{ Aglomerado 3} \\
\hline & $f$ & $\%$ & $f$ & $\%$ & $f$ & $\%$ \\
\hline Variaveis & & & & & & \\
\hline Sexo masculino & 10 & 91 & 2 & 25 & 8 & 67 \\
\hline Negros e/ou pardos & 8 & 73 & 5 & 62 & 12 & 100 \\
\hline Freqüência à escola 97 & 9 & 82 & 6 & 75 & 8 & 67 \\
\hline Não freqüência à escola 98 & 2 & 18 & 0 & 0 & 5 & 42 \\
\hline Não alfabetizado & 0 & 0 & 0 & 0 & 3 & 25 \\
\hline Participação de programa & 2 & 18 & 1 & 12 & 9 & 75 \\
\hline Usa drogas & 2 & 18 & 1 & 12 & 7 & 58 \\
\hline
\end{tabular}

consideramos como explicativos deste grupo. Detalhando cada grupo encontramos os seguintes dados (Tabela 5):

- Aglomerado 1: chamado de "integrados", com 11 participantes $(35,5 \%)$, encontramos $86 \%$ dos membros do sexo masculino, praticamente todos na escola $(82 \%)$, $100 \%$ deles alfabetizados e baixa participação em programas e uso de drogas $(18 \%)$.

- Aglomerado 2: chamado de "integradas", com 8 participantes $(25,8 \%)$ e diferenciando-se, em relação ao Aglomerado 1, em função do sexo, com predomínio do feminino $(75 \%)$ e freqüência à escola em 1998, com 100\%.

- Aglomerado 3: chamado de "risco", com 12 participantes $(38,7 \%)$, predomínio de negros e pardos $(100 \%)$, maioria do sexo masculino (67\%), menor freqüência à escola $(58 \%)$, maior participação em programas $(75 \%)$ e uso de drogas (58\%).

Quanto à representatividade dos participantes, consideramos que foram entrevistados praticamente todas as crianças e adolescentes que estavam em situação de rua na época do censo, que formaram o grupo "Rua". O mesmo não pode ser dito em relação ao grupo "Bairro", formado de moradores de apenas dois bairros de classe pobre, que foram construídos sob a forma de conjuntos habitacionais financiados pelo governo. Estes participantes foram alcançados por entrevistadores que moravam nestes bairros e tinham acesso a estes jovens.

O último ponto a ser analisado são os participantes que tinham sido entrevistados no Censo de 1994/95 (Martins, 1996a) e que foram também cadastrados neste Censo. Nesse estudo foram cadastrados 131 participantes, sendo 83 na categoria "Rua" e 48 na "Bairro". A categoria "Rua" foi dividida em quatro tipos, de acordo com o uso do espaço da rua (busca de renda, local de alimentação, de dormir e vínculo familiar).

Das 67 crianças e adolescentes entrevistadas nesta segunda fase do Censo encontramos 7 participantes que já tínhamos tido contato na primeira fase. Destes 7, na época, 6 eram crianças e uma adolescente. Quatro foram classificados como "tipo 1" (busca de renda para família, alimentação durante o dia na rua, local de dormir em casa e vínculo familiar estável) e 3 como "tipo 4" (busca de renda para sustento próprio, alimentação e local de dormir na rua e vínculo familiar instável ou ausente).

No cadastro de 1998, 2 continuam na escola (são irmãos gêmeos), defasados de série, mas alfabetizados e não usam drogas. Usam o espaço de rua para pedir e ter renda para seu sustento e ajudar em casa (têm vínculo familiar estável). Dos outros 5 participantes, 1 nunca foi à escola e os outros 4 saíram antes de concluírem a $2^{\text {a }}$ série do ensino fundamental, são analfabetos, e coincidentemente todos fazem uso de drogas e não têm vínculo familiar.

\section{Discussão}

Esta discussão procurará refletir os resultados obtidos neste censo em relação ao primeiro censo, estudos na área e os resultados em si deste estudo. No primeiro estudo (Martins, 1996a) foram entrevistadas 131 crianças e adolescentes, sendo 83 equivalentes ao nosso grupo "Rua" e 48 ao grupo "Bairro". Numa primeira comparação, temos uma redução no número total, em ambos os grupos, de 83 para 67 pessoas no "Rua", e de 48 para 31 no "Bairro", com diminuição de 19,3\% no primeiro grupo e $35,4 \%$ no segundo, mas olhando com mais profundidade, no primeiro censo, dos 83 participantes, 59 eram equivalentes ao Aglomerado 3 Trabalhadores, que correspondia a $71 \%$ do total e atualmente representam apenas $43,3 \%$, uma redução de $27,7 \%$.

Os tipos 2 e 3 do primeiro estudo se comparariam ao Aglomerado 2 - Desligando-se, onde tínhamos 15 crianças/ adolescentes (18\%) passaram para 11, correspondendo a $16,4 \%$ do total, mostrando uma ligeira diminuição. Quanto 
aos classificados como tipo 4, existiam apenas 9 participantes $(10,8 \%)$, que passaram para 27 casos, representando $40,3 \%$ do total, com um aumento de $66,6 \%$. Este último resultado pode ter pelo menos duas explicações. A primeira seria a própria forma de classificação usada, e como algumas das variáveis levantadas neste estudo não foram levantadas no primeiro censo, não é possível aplicarmos esta metodologia para aqueles dados. A segunda seria um aumento real deste tipo de criança nas ruas a partir do ano de 1997, quando houve uma mudança na política de atendimento da cidade, com o fechamento da Casa da Criança e o progressivo desativamento do Projeto Santa Mônica (Martins, 2000).

No primeiro censo, entrevistamos 83 crianças/ adolescentes, e destes somente 15 estariam com mais de 18 anos em 1998, o que os tornariam não elegíveis para este estudo. Deste universo ficaram 68 participantes que poderiam ainda estar em situação de rua, mas só foram entrevistados, neste estudo, sete destes participantes, que atualmente são adolescentes e permanecem em situação de rua. Este número nos mostra que passados 3 anos 18\% deixaram a situação de adolescente e se tornam adultos e dos $82 \%$ restantes somente $10,3 \%$ permaneceram nesta situação.

Revendo estudos da área o primeiro a ser comparado é o de Lima (1992), com crianças e adolescentes que perambulavam pelo centro da cidade, foco desta pesquisa. Nesse estudo foram entrevistados somente os usuários de cola de sapateiro, que formavam um grupo de 20 pessoas. Como no primeiro censo não levantamos especificamente o uso de drogas, não temos este dado, mas neste estudo encontramos, no grupo "Rua", usando o mesmo espaço do estudo de Lima, 32 participantes fazendo uso não só de cola de sapateiro como de outras drogas (crack, maconha e thinner), mostrando um aumento $37 \%$ neste grupo.

Todos os estudos posteriores ao do censo 1994/95 procuram conhecer aspectos particulares destas crianças e adolescentes, sem uma preocupação de levantamento geral da situação, em cada cidade onde foram feitos. Entre os temas pesquisados temos o da construção da identidade (Bonamigo, 1996; Graciani, 1992), conduta sexual (Hutz \& Forster, 1996), características das crianças trabalhadoras (Maciel, Brito \& Camino, 1997; Santos e Silva \& cols., 1998), compreensão das desigualdades sociais (Maciel, Brito \& Camino, 1998), representações sociais (Alves-Mazzotti, 1997) e estudos sobre o uso de drogas (Noto, Nappo, Galduróz, Mattei \& Carlini, 1997; Rigon \& Ramanelli, 2000).

O estudo de Noto e colaboradores (1997), realizado em seis capitais brasileiras, teve como participantes crianças e adolescentes em situação de rua, mas vinculados a algum tipo de organização, governamental ou não.
Embora esta amostra possa não ser representativa da população de rua, não temos dúvidas que os dados sobre consumo de drogas sejam alarmantes. No grupo "Rua" encontramos 40 crianças e/ou adolescentes que já participaram de algum programa assistencial, representando $59,7 \%$ dos entrevistados e 32 que já usaram ou estão usando algum tipo de droga (47,8\%). Quando comparados com os $78,1 \%$ dos participantes com uso recente na cidade de São Paulo notamos uma grande discrepância, o que pode estar mostrando um perfil diferenciado para os que vivem em cidades do interior.

Finalmente, olhando os dados atuais em si, com o uso da análise de aglomerados (Cluster Analysis) para uma classificação mais clara, com a utilização do número de atributos que parecem ser discriminantes da situação de rua, não ficando na tradicional situação de usar uma ou duas variáveis, como vínculo familiar e/ou tipo de atividade exercida na rua, que produzem tipologias do tipo tudo ou nada e agrupam crianças e adolescentes com características diferenciadas. Os aglomerados resultantes parecem diferenciar claramente três grupos distintos: solitários, desligando-se e trabalhadores.

Os solitários correspondem ao tradicional "criança de rua", com seus membros sendo adolescentes do sexo masculino, metade de negros ou pardos, tempo de rua alto, abandono da escola, não alfabetizados, procedentes da própria cidade, participação em vários programas de atendimento, usando o espaço de rua para mendigar e usar drogas e com retorno irregular ou não freqüente para casa. A permanência destas pessoas na situação de rua, apesar de serem atendidas em programas de reinserção social, mostra que as formas de atuação destes projetos não têm sido adequadas e precisam ser repensadas, como por exemplo não exigir retorno imediato à escola, da qual foram expulsos ou saíram por "não gostar" ou "dificuldade de aprendizagem". Os programas devem inicialmente alfabetizar estas pessoas.

O segundo aglomerado, dos "desligando-se" mostra um pequeno grupo em transição da situação de "trabalhadores" para o dos "solitários", com um abandono progressivo da escola, aumento no uso de drogas e não retorno diário à família. Este grupo precisa ser mantido na escola e ter sua família com condições mínimas de sobrevivência. Em outro estudo mostramos o impacto positivo deste tipo de apoio e o que ocorre quando este é retirado (Martins, 2000).

O último aglomerado, dos “trabalhadores”, se caracteriza por mais da metade deles usarem o espaço de rua com alguma forma de trabalho (engraxates, ambulantes ou panfleteiros), maioria branca, permanência na escola, não uso de drogas e retorno diário para a família. Da mesma forma que no aglomerado anterior programas de 
atendimento voltados para o fortalecimento do núcleo familiar são muito positivos e de custo relativamente baixo, principalmente quando comparado com o atendimento aos do aglomerado dos "solitários", e principalmente aos que acabam enveredando pelos atos infracionais e são colocados em sistema de internato.

Finalizamos este artigo, salientando a necessidade de um conhecimento mais detalhado dos participantes de cada grupo, numa abordagem qualitativa, para preenchermos as lacunas sobre a realidade social, do ponto de vista deles. Principalmente, suas relações com a escola e o que, ou como, se constituem suas famílias.

\section{Referências}

Alves-Mazzotti, A. J. (1997). Representações sociais de meninos de rua. Educação e Realidade, 22(1),183-207.

Amado, J. (1965). Capitães da areia. São Paulo: Martins.

Aptekar, L. (1991). Are colombian street children neglected? The contribuitions of ethnographic and ethnohistorical approaches to the study of children. Anthropology \& Education Quarterly, 22, 326-349.

Aptekar, L. (1996). Crianças de rua nos países em desenvolvimento: Uma revisão de suas condições. Psicologia: Reflexão e Crítica, 9, 153-184.

Bonamigo, L. R. (1996). O trabalho e a construção da identidade: Um estudo sobre meninos trabalhadores na rua. Psicologia: Reflexão e Crítica, 9,129-152.

Carrizosa, S. O. de \& Poertner, J. (1992). Latin American street children: Problem, programmes and critique. International Social Work, 35, 405413.

CONDECA (1996). Estatuto da Criança e do Adolescente. São Paulo: Imprensa Oficial do Estado.

Cosgrove, J. G. (1990). Towards a working definition of street children. International Social Work, 33,185-192.

Danseco, E. R. \& Holden, E. W. (1998). Are there different types of homeless families? A typology of homeless families based on cluster analysis. Family Relations, 47(2), 159-65.

Dickens, C. (1965). The adventures of Oliver Twist. London, New York: Oxford University.

Ferreira, R. M. F. (s.d.). Meninos de rua. Expectativas, valores de menores marginalizados em São Paulo. São Paulo: Ibrex.

Geremek, B. (1995). Os filhos de Caim. São Paulo: Companhia das Letras.

Graciani, M. S. S. (1992). A construção social da identidade de meninos (as) de rua. Revista Brasileira de Crescimento e Desenvolvimento Humano, 2(1), $147-153$.

Hair, J. F., Anderson, R. E., Tatham, R. L. \& Black, W. C. (1998). Multivariate data analysis: With readings. Englewood Cliffs: Prentice Hall.

Hutz, C. \& Forster, L. M. K. (1996). Comportamento e atitudes sexuais de crianças de rua. Psicologia: Reflexão e Crítica, 9, 209-229.

Lima, G. (1992). Meninos de rua em São José do Rio Preto, Estado de São Paulo. Universitas, 2(1), 41-44.

Lusk, M. W. (1992). Street children of Rio de Janeiro. International Social Work, 35, 293-305.

Maciel, C., Brito, S. \& Camino, L. (1997). Caracterização dos meninos em situação de rua de João Pessoa. Psicologia: Reflexão e Crítica, 10, 315-334.

Maciel, C., Brito, S. \& Camino, L. (1998). Caracterização dos meninos em situação de rua de João Pessoa. Psicologia: Reflexão e Crítica. 11, 209-232.
Manzato, A. J. (1983). Análise bierárquica de agrupamentos para distribuiçoes multinomiais. Dissertação de Mestrado não-publicada, Curso de PósGraduação em Estatística, Instituto de Matemática e Estatística, Universidade de São Paulo. São Paulo, SP.

Martins, R. A. (1996a). Censo de crianças e adolescentes em situação de rua em São José do Rio Preto. Psicologia: Reflexão e Crítica, 9, 101-122.

Martins, R. A. (1996b). Crianças e adolescentes em situação de rua: Definições, evoluçôs e políticas de atendimentos. Em S. H. Koller (Org.), Aplicações da psicologia na melhoria de qualidade de vida (pp. 35-44). Porto Alegre: ANPEP.

Martins, R. A. (2000). Programa de apoio sociofamiliar a crianças e adolescentes em situação de rua. Nuances, 6, 193-201.

Mermet, J. (1995). Bibliography on street cbildren. Geneve: Henry Dunant Institute.

Noto, A. R, Nappo, S., Galduróz, J. C. F., Mattei, R. \& Carlini, E. A. (1997). IV Levantamento sobre o uso de drogas entre crianças e adolescentes em situação de rua de seis capitais brasileiras. São Paulo: AFIP e CONFEN.

Oliveira, W. de, Baizerman, M. \& Pellet, L. (1992). Street children in Brazil and their helpers: Comparative views on aspirations and the future. International Social Work, 35, 163-176.

Pereira, J. C. R. (1999). Análise de dados qualitativos: Estratégias metodológicas para as ciências da saúde, bumanas e sociais. São Paulo: Edusp e Fapesp.

Pereira, J. C. R. \& Saes, S. G. (1995). Avaliação de estratégias de gestão de ciência e tecnologia: Um estudo de caso. Revista de Saúde Pública, 29, 308-317.

Pilotti, F. (1995). Crise e perspectivas da assistência à infância na América Latina. Em F. Pilotti \& I. Rizzini (Orgs.), $A$ arte de governar crianças: $A$ história das políticas sociais, da legislação e da assistência à infância no Brasil (pp.11-45). Rio de Janeiro: Instituto Interamericano Del Niño, Editora Universitária Santa Úrsula, Amais.

Rigon, J. M. \& Romanelli, G. (2000). Adolescentes em situação de rua e consumo de crack em Ribeirão Preto. Em Seminário de Pesquisa, III, (Tomo II, pp.45-49). Ribeirão Preto: Programa de Pós- Graduação em Psicologia da Faculdade de Filosofia, Ciências e Letras de Ribeirão Preto, Universidade de São Paulo.

Rizzini, I. \& Rizzini, I. (1996). "Menores" institucionalizados e meninos de rua. Em A. Fausto \& R. Cervini (Orgs.), O trabalho e a rua: Crianças e adolescentes no Brasil urbano dos anos 80 (2 $2^{\mathrm{a}}$ ed., pp. 69-90). São Paulo: Cortez.

Romesburg, C. H. (1990). Cluster analysis for researchers. Malabar, Florida: R. E. Krieger.

Rosemberg, F. (1994). Contagem de crianças e adolescentes em situação de rua na cidade de São Paulo. Relatório Técnico, Secretaria da Criança, Família e Bem-Estar Social. São Paulo.

Santos, A., Silva, A., Reppold, C. T., Santos, C. L., Prade, L. T., Silva, M. R., Alves, P. B. \& Koller, S. H. (1998). Crianças em situação de rua de Porto Alegre: Um estudo descritivo. Psicologia: Reflexão e Crítica, 11, 441-447.

UNICEF - United Nations Children's Fund (1991). The state of the world's children. Oxford: Oxford University.

UNICEF - United Nations Children's Fund (1993). The state of the world's children. Oxford: Oxford University.

Valladares, L. P. (Org.) (1988). A "infância pobre" no Brasil. Uma análise da literatura, da ação e das estatisticas. Relatório de Convênio Fundação Ford/ IUPERJ. Rio de Janeiro: IUPERJ.

Williams, C. (1993). Who are "street children?" A hierarchy of street use and appropriate responses. Child Abuse \& Neglect, 17, 831-841.

Recebido: 14/02/2001 Revisado: 08/08/2001 Aceito: $17 / 10 / 2001$

\section{Sobre o autor}

Raul Aragão Martins é Psicólogo, Doutor em Psicologia pela FGV (Rio de Janeiro), Professor de Psicologia da Educação no Instituto de Biociências, Letras e Ciências Exatas e do Programa de PósGraduação em Educação da Faculdade de Filosofia e Ciências na Universidade Estadual Paulista (UNESP), Campus de São José do Rio Preto. 